\title{
Descending Rational Points on Elliptic Curves to Smaller Fields
}

\author{
Amir Akbary * and V. Kumar Murty ${ }^{\dagger}$
}

\begin{abstract}
In this paper, we study the Mordell-Weil group of an elliptic curve as a Galois module. We consider an elliptic curve $E$ defined over a number field $K$ whose Mordell-Weil rank over a Galois extension $F$ is 1,2 or 3 . We show that $E$ acquires a point (points) of infinite order over a field whose Galois group is one of $C_{n} \times C_{m}$ $(n=1,2,3,4,6, m=1,2), D_{n} \times C_{m}(n=2,3,4,6, m=1,2), A_{4} \times C_{m}$ $(m=1,2), S_{4} \times C_{m}(m=1,2)$. Next, we consider the case where $E$ has complex multiplication by the ring of integers $\mathcal{O}$ of an imaginary quadratic field $\mathfrak{K}$ contained in $K$. Suppose that the $\mathcal{O}$-rank over a Galois extension $F$ is 1 or 2 . If $\mathfrak{K} \neq \mathbb{Q}(\sqrt{-1})$ and $\mathbb{Q}(\sqrt{-3})$ and $h_{\mathfrak{K}}$ (class number of $\mathfrak{K}$ ) is odd, we show that $E$ acquires positive $\mathcal{O}$-rank over a cyclic extension of $K$ or over a field whose Galois group is one of $\mathrm{SL}_{2}(\mathbb{Z} / 3 \mathbb{Z})$, an extension of $\mathrm{SL}_{2}(\mathbb{Z} / 3 \mathbb{Z})$ by $\mathbb{Z} / 2 \mathbb{Z}$, or a central extension by the dihedral group. Finally, we discuss the relation of the above results to the vanishing of $L$-functions.
\end{abstract}

Table of Contents

1. Introduction

2. The minimal subfield

3. Group theoretic lemmas

4. $E(F)$ of $\mathbb{Z}$-rank $1,2,3$ or $\mathcal{O}$-rank 1 or 2

5. Vanishing of $L$-functions

6. Elliptic analogue of Stark's theorem

\section{Introduction}

Let $E$ be an elliptic curve defined over a number field $K$. By the Mordell-Weil theorem, the group $E(K)$ of points of $E$ with coordinates in $K$ is finitely generated. We write $\operatorname{rank}(E(K))$ for the rank of $E(K)$ modulo torsion. Let $F$ be a finite Galois extension

${ }^{*}$ Research partially supported by Concordia Research Funds.

${ }^{\dagger}$ Research partially supported by a grant from NSERC. 
of $K$ with group $G$. In this paper, we consider the Mordell-Weil group $E(F)$ as a $\mathbb{Z}[G]$ module. Since the torsion subgroup $E(F)_{\text {tors }}$ has been extensively studied (see for example, Serre [20]), we shall restrict ourselves to the free part of $E(F)$. The question of studying this as a Galois module was raised in the works of Mazur [9], Mazur and Swinnerton-Dyer [10], Coates and Wiles [3] Rohrlich [16], and [17], to name a few.

Philosophically, it is of interest to note one basic difference between the free part and the torsion part as Galois modules. For example, consider the Galois module of $\ell$-torsion points $E[\ell]$. The field $K(E[\ell])$ obtained by adjoining the coordinates of points in $E[\ell]$ has Galois group contained in $\operatorname{Aut}(E[\ell]) \simeq \mathrm{GL}_{2}(\mathbb{Z} / \ell)$. Serre's theorem tells us that if $E$ is without complex multiplication, then for large $\ell$, it is in fact equal to $\mathrm{GL}_{2}(\mathbb{Z} / \ell)$. On the other hand, let $K\left(E(F)_{\text {free }}\right)$ be the field generated by adjoining the coordinates of any free $\mathbb{Z}[\operatorname{Gal}(F / K)]$-submodule of $E(F) \otimes \mathbb{Q}$ to $K$ and suppose that $\operatorname{rank}(E(F))=r$, then $\operatorname{Gal}\left(K\left(E(F)_{\text {free }}\right) / K\right)$ is conjugate to a subgroup of $\mathrm{GL}_{r}(\mathbb{Z})$. This imposes two restrictions on this Galois group. Firstly, by Jordan's theorem, a finite subgroup of $\mathrm{GL}_{r}(\mathbb{C})$ has a normal Abelian subgroup of index bounded by a function of $r$ alone. Secondly, this is an integral representation. By the work of Nori [14], there are many restrictions on the finite subgroups of $\mathrm{GL}_{r}(\mathbb{Z})$. Another restriction imposed on these Galois groups arises from the fact that the height pairing on the Mordell-Weil group is respected by the action of Galois.

In another direction, there is the connection with the $L$ function of the elliptic curve. A well known theorem of Coates and Wiles [3] for CM elliptic curves asserts that if $E(K)$ is infinite, then the $L$-function $L(E / K, s)$ vanishes at $s=1$. From the work of Kolyvagin [7], a similar result is known for (modular) elliptic curves over $\mathbb{Q}$. This is in accordance with the general conjecture of Birch and Swinnerton-Dyer. Here, we shall discuss the following:

Problem 1: Let $F / K$ be a finite Galois extension. If $E(F)$ is infinite, does $L(E / F, s)$ vanish at $s=1$ ?

Since the extensions of Coates-Wiles and Kolyvagin theorems to Abelian extensions are known (due respectively to Arthaud [1], and Rubin [18] in the CM-case and Kato (unpublished) in the modular case), we will show that the existence of an Abelian subextension $M$ of $F / K$ with $E(M)$ infinite implies a positive answer to Problem 1 (see Theorem 4). So we shall consider the following related problem.

Problem 2: Let $F / K$ be a finite Galois extension. If $E(F)$ is infinite, then under what conditions can we produce an Abelian subextension $M$ of $K(K \subseteq M \subseteq F)$ such that $E(M)$ is infinite?

We wish to draw the analogy of this question with a result of Stark [22] for Artin $L$-functions. He shows that if $F / K$ is Galois and the Dedekind zeta function $\zeta_{F}(s)$ has a simple zero at a point $s=s_{0}$, then there is a subextension $K \subseteq M \subseteq F$ with the property that $\zeta_{M}\left(s_{0}\right)=0$ and $M / K$ is Abelian (in fact, cyclic). Moreover, if $N$ is any other subfield satisfying $\zeta_{N}\left(s_{0}\right)=0$, we must have $M \subseteq N$. 
In section 4 , we consider an elliptic curve $E$ defined over $K$ whose Mordell-Weil rank over a Galois extension $F$ is 1 or 2 . If the rank of $E(F)$ is one, we observe (Theorem 1, (i)) that a Stark type result holds here. If the rank of $E(F)$ is two, we show that $E$ acquires two points of infinite order over a cyclic extension of $K$ with Galois group $C_{n}(n=1,2,3,4,6)$ contained in $F$ or over a dihedral extension with Galois group $D_{n}(n=2,3,4,6)$. Then we establish a similar result in the rank three case (Theorem 1, (iii)). In the case that $E$ has complex multiplication, we can also study the Mordell-Weil group $E(F)$ as an $\mathcal{O}[G]$-module. Here $E$ has complex multiplication by the ring of integers $\mathcal{O}$ of an imaginary quadratic field $\mathfrak{K}$ contained in $K$. We are able to establish the analogues of the above results in the case that $E(F)$ has $\mathcal{O}$-rank 1 or 2 (Theorems 2 and 3 ).

In the final section, by considering the order of vanishing of the $L$-function of $E$ at a point $s=\omega$, we investigate some analytic analogues of our results in section 4 . In the case of a simple zero, we prove an analogue of Stark's theorem for a certain class of elliptic curves (Corollary 1). Also, by analogy with [13], we formulate a statement for higher order zeros but it would depend on the holomorphy of the $L$-functions obtained by twisting the $L$-function of $E$ with certain Artin characters (see Proposition 6).

It is clear that much work remains to be done to elucidate the Galois module structure of the Mordell-Weil group. We hope that the explicit results of this paper may help in this effort.

Acknowledgement: We would like to thank Henri Darmon and Hershy Kisilevsky for several helpful discussions. This paper was started during the CRM workshop at St. Adele in 1992, and completed while the second author was visiting the CRM during the special year on Arithmetic Algebraic Geometry in 1998-1999. He thanks the CRM for its hospitality.

\section{The minimal subfield}

Definition: Let $E$ be an elliptic curve defined over $K$ and let $F / K$ be an extension (not necessarily Galois) of number fields. Suppose that $\operatorname{rank}(E(F))=r$, then the minimal subfield $F_{r}$ is a subfield with $K \subseteq F_{r} \subseteq F$, such that

(i) $\operatorname{rank}\left(E\left(F_{r}\right)\right)=\operatorname{rank}(E(F))$.

(ii) If $K \subseteq M \subseteq F$ and $\operatorname{rank}(E(M))=\operatorname{rank}(E(F))$, then $F_{r} \subseteq M$.

Proposition 1 For any finite extension $F / K$ and elliptic curve $E$ defined over $K$ with $\operatorname{rank}(E(F))=r, F_{r}$ exists and is unique. Also, if $F / K$ is Galois then $F_{r} / K$ is Galois.

Proof: We need only prove that if $K \subseteq M_{1}, M_{2} \subseteq F$ are subfields such that

$$
\operatorname{rank}\left(E\left(M_{1}\right)\right)=\operatorname{rank}\left(E\left(M_{2}\right)\right)=r
$$

then

$$
\operatorname{rank}\left(E\left(M_{1} \cap M_{2}\right)\right)=r .
$$


Indeed, $E\left(M_{1}\right) \otimes \mathbb{Q}=E\left(M_{2}\right) \otimes \mathbb{Q}$. Hence, there is a lattice $L$ contained in $E\left(M_{1}\right) \cap$ $E\left(M_{2}\right)$ which is of finite index in both $E\left(M_{1}\right)$ and $E\left(M_{2}\right)$. But then $L$ is fixed by $\operatorname{Gal}\left(\tilde{F} / M_{1}\right)$ and by $\operatorname{Gal}\left(\tilde{F} / M_{2}\right)$ where $\tilde{F}$ is the normal closure of $F / K$. Thus, it is fixed by $\operatorname{Gal}\left(\tilde{F} /\left(M_{1} \cap M_{2}\right)\right)$ and so it is contained in $E\left(M_{1} \cap M_{2}\right)$. Thus the rank of $E\left(M_{1} \cap M_{2}\right)$ is $r$ as claimed.

If $F / K$ is Galois, we can apply this argument to $M$ and a conjugate of $M$, and from this, we see that the minimal subfield is necessarily Galois over $K$.

Now we give another description of the minimal subfield. Let $F / K$ be a finite Galois extension, then since $\operatorname{Gal}(F / K)$ acts on $E(F) \otimes \mathbb{Q}$, we have a representation

$$
\rho: \operatorname{Gal}(F / K) \rightarrow \operatorname{Aut}(E(F) \otimes \mathbb{Q}) \simeq \mathrm{GL}_{r}(\mathbb{Q})
$$

where $\operatorname{rank}(E(F))=r$. Then, there exists a free submodule of $E(F) \otimes \mathbb{Q}$ of rank $r$ on which $\operatorname{Gal}(F / K)$ acts. For example, if $m=\left|E(F)_{\text {tors }}\right|$, then we can take $m E(F)$. Each such submodule $X$ (say) gives a representation

$$
\rho_{X}: \operatorname{Gal}(F / K) \rightarrow \operatorname{Aut}(X) \simeq \mathrm{GL}_{r}(\mathbb{Z}) .
$$

Moreover, different choices of $X$ yield representations isomorphic over $\mathbb{Q}$. In particular, $\operatorname{Ker}\left(\rho_{X}\right)$ is equal to $\operatorname{Ker}(\rho)$ and is independent of $X$. Thus, the field $K(X)$ obtained by adjoining the coordinates of points in $X$ to $K$ is independent of the choice of $X$. We denote this field by $K\left(E(F)_{\text {free }}\right)$.

Proposition 2 Let $F / K$ be a finite Galois extension. If $\operatorname{rank}(E(F))=r \geq 1$, then (i) there is a subextension $M$, Galois over $K$ such that $E(M) \otimes \mathbb{Q}=E(F) \otimes \mathbb{Q}$ and the representation

$$
\rho_{f}: \operatorname{Gal}(M / K) \rightarrow \operatorname{Aut}(E(M) \otimes \mathbb{Q})
$$

is faithful. Moreover, $\operatorname{Im}\left(\rho_{f}\right)$ is conjugate to a finite subgroup of $\mathrm{GL}_{r}(\mathbb{Z})$.

(ii) $M=K\left(E(F)_{\text {free }}\right)$.

(iii) $M$ is the minimal subfield defined in the beginning of the section.

Proof: (i) Suppose that $\rho$ is the representation of $\operatorname{Gal}(F / K)$ in $E(F) \otimes \mathbb{Q}$. Let $M$ be the fixed field of ker $\rho$. Since

$$
(E(F) \otimes \mathbb{Q})^{\operatorname{Ker} \rho}=(E(F) \otimes \mathbb{Q})^{\operatorname{Gal}(F / M)}=E(M) \otimes \mathbb{Q}
$$

(see [16], p. 126) and since $M$ is the fixed field of $k e r \rho, \operatorname{Gal}(F / M)$ acts trivially on $E(F) \otimes \mathbb{Q}$. This shows that $E(F) \otimes \mathbb{Q}=E(M) \otimes \mathbb{Q}$ and $\rho_{f}$ is faithful. The argument before the proposition shows that $\operatorname{Im}\left(\rho_{f}\right)$ is conjugate to a finite subgroup of $\mathrm{GL}_{r}(\mathbb{Z})$.

(ii) This is clear from the argument before the proposition.

(iii) Let $K \subseteq L \subseteq F$ and $\operatorname{rank}(E(L))=\operatorname{rank}(E(F))$, then from the proof of Proposition 1, we know that $\operatorname{rank}(E(L \cap M))=\operatorname{rank}(E(M))$ and $E(M) \otimes \mathbb{Q}=$ $E(L \cap M) \otimes \mathbb{Q}$. This shows that $\operatorname{Gal}(M /(L \cap M))$ acts trivially on $E(M) \otimes \mathbb{Q}$ and therefore it is contained in the kernel of the representation $\rho_{f}$. But $k e r \rho_{f}=\{i d\}$, which implies that $\operatorname{Gal}(M /(L \cap M))=\{i d\}$. Thus $L \cap M=M$ and therefore $M \subseteq L$. This proves that $M$ is the minimal subfield. 
Proposition 3 Let $F / K$ be a finite Galois extension, then the degree of the minimal subfield $F_{r}$ over $K$ is bounded as a function of $r$ alone.

Proof: By Proposition 2, we can consider $\operatorname{Gal}\left(F_{r} / K\right)$ as a finite subgroup of $\mathrm{GL}_{r}(\mathbb{Z})$ (and therefore $\mathrm{GL}_{r}(\mathbb{C})$ ). By Jordan's theorem a finite subgroup of $\mathrm{GL}_{r}(\mathbb{C}$ ) has a normal Abelian subgroup $G_{1}$ whose index is bounded by a function of $r$ alone. So it is enough to prove that the order of $G_{1}$ is bounded by a function of $r$ alone.

Now, let $L$ be the fixed field of $G_{1}$ in $F_{r} / K$, and let $\rho_{1}$ be the restriction of the representation $\rho_{f}$ (defined in Proposition 2) to $G_{1}=\operatorname{Gal}\left(F_{r} / L\right)$. Then

$$
\rho_{1}=\psi_{1} \oplus \psi_{2} \oplus \ldots \oplus \psi_{r}
$$

where $\psi_{i}$ 's are one dimensional characters of $G_{1}$. Since the values of the $\psi_{i}$ satisfy a degree $r$ polynomial over $\mathbb{Q}$, if $\psi_{i}$ takes values in $\mathbb{Q}\left(\zeta_{m_{i}}\right)$, we must have $\phi\left(m_{i}\right) \leq r$. Since $\rho_{1}$ is faithful, this implies that the order of $G_{1}$ is bounded by a function of $r$ alone.

\section{Group theoretic lemmas}

In this section, we collect some group theoretic results which will be needed in the sequel.

Lemma 1 Let the representation $\rho: G \rightarrow \mathrm{GL}_{2}(\mathbb{Z})$ be faithful, then

(i) if $\rho$ is reducible, $G$ is cyclic $C_{n}(n=1,2,3,4,6)$ or $G \simeq \mathbb{Z} / 2 \mathbb{Z} \oplus \mathbb{Z} / 2 \mathbb{Z} \simeq D_{2}$.

(ii) if $\rho$ is irreducible, $G$ is dihedral $D_{n}(n=3,4,6)$.

Proof: (i) Suppose that $\rho$ is reducible. Let $\chi$ be the character of $\rho$. Then $\chi=\psi_{1}+\psi_{2}$ over $\mathbb{C}$, where $\psi_{1}$ and $\psi_{2}$ are one dimensional characters of $G$. As the characteristic polynomial of $\rho$ has coefficients in $\mathbb{Z}$, we must have $\psi_{1}=\overline{\psi_{2}}$ or $\psi_{1}$ and $\psi_{2}$ characters of order 2 . Since $\rho$ is faithful, in the latter case, $G \simeq \mathbb{Z} / 2$ or $G \simeq \mathbb{Z} / 2 \oplus \mathbb{Z} / 2 \simeq D_{2}$ and in the former case, $G$ is cyclic.

Now if $r$ is a generator of the cyclic group $G$ and $\operatorname{ord}(r)=n$, then $\rho(r)$ is conjugate to a diagonal matrix over $\mathbb{C}$ like

$$
\left(\begin{array}{cc}
e^{\frac{2 \pi i h}{n}} & 0 \\
0 & e^{-\frac{2 \pi i h}{n}}
\end{array}\right)
$$

where $0 \leq h<n$ and $(h, n)=1$. Here, $e^{\frac{2 \pi i h}{n}}$ is a primitive $n$-th root of unity which is also a root of a quadratic polynomial over $\mathbb{Z}$ (i.e. the characteristic polynomial of the above matrix). Therefore $\phi(n)=\left[\mathbb{Q}\left(e^{\frac{2 \pi i h}{n}}\right): \mathbb{Q}\right] \leq 2$ and so $n=1,2,3,4,6$.

(ii) Since $\rho$ is faithful, we can consider $G$ as a finite subgroup of $G_{2}(\mathbb{R})$. We know that a finite subgroup of $\mathrm{GL}_{2}(\mathbb{R})$ is conjugate to a subgroup of $\mathrm{O}_{2}(\mathbb{R})$ and is therefore cyclic or dihedral (see [15], p. 22, Theorem 9). As $\rho$ is irreducible, $G \simeq D_{n}=$ 
$\left\langle r, s ; r^{n}=1, s^{2}=1\right.$, srs $\left.=r^{-1}\right\rangle$. Let $H=\langle r\rangle$, then $\left.\chi\right|_{H}=\psi_{1}+\psi_{2}$ over $\mathbb{C}$, where $\psi_{1}(r)=e^{\frac{2 \pi i h}{n}}$ and $\psi_{2}(r)=e^{-\frac{2 \pi i h}{n}}$ (see [19], p. 37), so by reasoning similar to part (i), $\operatorname{ord}(H)=n=1,2,3,4,6$. Moreover, $n \neq 1,2$ since in these cases $D_{n}$ is Abelian.

Let $H_{1}$ and $H_{2}$ be subgroups of a group $G$ and let $x \in G$. Set

$$
J\left(H_{1}, H_{2}, x\right)=H_{2} \cup\left\{x g \mid g \in H_{1}, g \notin H_{2}\right\} .
$$

Lemma 2 Let $H_{1}$ and $H_{2}$ be subgroups of a group $G$ such that $H_{2} \subset H_{1}$ and $\left[H_{1}\right.$ : $\left.H_{2}\right]=2$. Let $x \in G-H_{2}$ be an element of order 2 which commutes with all elements of $H_{1}$. Then

(i) $J\left(H_{1}, H_{2}, x\right)$ is a subgroup of $G$.

(ii) $H_{1} \simeq H_{2} \times C_{2}$ if $x \in H_{1}$.

(iii) $H_{1} \simeq J\left(H_{1}, H_{2}, x\right)$ if $x \notin H_{1}$.

Proof: It is straightforward.

Lemma 3 Let the representation $\rho: G \rightarrow \mathrm{GL}_{3}(\mathbb{Z})$ be faithful, then $G$ is isomorphic to one of the following:

$$
C_{n} \times C_{m}, \quad D_{p} \times C_{m}, \quad A_{4} \times C_{m}, \quad S_{4} \times C_{m}
$$

where $n=1,2,3,4,6, p=2,3,4,6$ and $m=1,2$.

Proof: Since $\rho$ is faithful we consider $G$ as a finite subgroup of $\mathrm{O}_{3}(\mathbb{R})$. First suppose that $G \subset \mathrm{SO}_{3}(\mathbb{R})$. Then it is known that $G$ is either cyclic, dihedral, $A_{4}, S_{4}$ or $A_{5}$ (see [15], p. 35, Theorem 11). Note that in this case if $A \in G$, then there is an orthonormal matrix $P$ such that

$$
P^{-1} A P=\left(\begin{array}{ccc}
\cos \alpha & -\sin \alpha & 0 \\
\sin \alpha & \cos \alpha & 0 \\
0 & 0 & 1
\end{array}\right)
$$

(see [15], p. 35, corollary 1 ), with $\operatorname{tr}\left(P^{-1} A P\right) \in \mathbb{Z}$. Therefore $2 \cos \alpha \in \mathbb{Z}$. It is easily seen from here that if $G \subset \mathrm{SO}_{3}(\mathbb{R})$, the order of any element of $G$ must be 2, 3, 4 or 6 , and therefore $G$ must be one of the following

$$
C_{n}(n=1,2,3,4,6), \quad D_{p}(p=2,3,4,6), \quad A_{4}, \quad S_{4} .
$$

Now suppose that $G \not \subset \mathrm{SO}_{3}(\mathbb{R})$. Let $G_{s}=G \cap \mathrm{SO}_{3}(\mathbb{R})$ and note that $-I$ ( $I$ is the identity matrix) is an element of order 2 in $\mathrm{O}_{3}(\mathbb{R})$ which is not in $G_{s}$ and it commutes with all elements of $G$. Therefore, by Lemma 2 , either $G \simeq G_{s} \times C_{2}$ or $G \simeq J\left(G, G_{s},-I\right) . G_{s}$ and $J\left(G, G_{s},-I\right)$ are finite subgroups of $\mathrm{SO}_{3}(\mathbb{R})$ and therefore they are in the list given in $(*)$. This completes the proof.

Now let $\mathcal{O}$ denote the ring of integers of an imaginary quadratic field $\mathfrak{K}$. We fix an embedding $\mathfrak{K} \hookrightarrow \mathbb{C}$.

Notation. We denote the center of a group $G$ by $C e n t(G)$. 
Lemma 4 Let $G$ be a group with a normal subgroup $H$ of prime index. Let $\rho: G \rightarrow$ $\mathrm{GL}_{2}(\mathcal{O})$ be a faithful and irreducible representation of $G$, and let $\chi$ be the character of $\rho$. Then

(i) either $\chi=\operatorname{Ind}_{H}^{G} \psi, \psi(1)=1$ or $\left.\chi\right|_{H}$ is irreducible.

In the case that $\chi=\operatorname{Ind}_{H}^{G} \psi, \psi(1)=1$, let us set $N=\operatorname{Ker} \psi$.

(ii) If $N=\{i d\}$, then $H \simeq C_{n}(n=2,3,4,6,8,12)$.

(iii) If $N \neq\{$ id $\}$ and $[G: H]=2$ then for all $\sigma \in G-H$ we have $N \cap \sigma^{-1} N \sigma=\{i d\}$.

Proof: (i) By Proposition 24 of [19] (p. 61), there exists a subgroup $J$ of $G$, unequal to $G$ and containing $H$ such that either $\chi=\operatorname{Ind}_{J}^{G} \psi, \psi(1)=1$ or $\left.\chi\right|_{J}$ is isotypic. Since $H$ has prime index in $G$ then $J=H$.

If $\left.\chi\right|_{H}$ is isotypic and reducible then $H \subset \operatorname{Cent}(G)$. But $G / H$ is cyclic and therefore $G / \operatorname{Cent}(G)$ is also cyclic. This implies that $G$ is Abelian which is a contradiction since $G$ has a two dimensional irreducible representation. The only other possibility is that $\left.\chi\right|_{H}$ is irreducible.

(ii) Since $\psi$ is faithful, $H$ is isomorphic to a finite subgroup of $\mathbb{C}^{\times}$and therefore is cyclic. A characteristic polynomial argument similar to the one in Lemma 1 shows that the order $n$, say, of this group can only be $2,3,4,5,6,8,10$ or $12(n \neq 1$, since $G$ cannot be Abelian). Since $H$ is cyclic, $\left.\chi\right|_{H}=\psi+\psi^{\prime}$.

Now if $n=5, \psi$ and $\psi^{\prime}$ take values in the group of 5-th roots of unity, and therefore $\left.\chi\right|_{H}$ takes values in $\mathbb{Q}\left(\zeta_{5}\right) \cap \mathfrak{K}=\mathbb{Q}$. The characteristic polynomial of $\left.\rho\right|_{H}$ has real coefficients and so either $\psi$ and $\psi^{\prime}$ are both real or $\psi^{\prime}$ is the complex conjugate of $\psi$. Since $\psi$ has order 5 , the first case cannot occur. Hence, we are in the second case, and this implies that the character $\left.\chi\right|_{H}$ takes values in $\mathbb{Q}\left(\zeta_{5}\right)^{+}$which is not $\mathbb{Q}$ and this is a contradiction. Therefore, $n \neq 5$. In a similar way, we can show that $n \neq 10$.

(iii) If $N \neq\{i d\}$ then $N$ cannot be normal in $G$. Indeed, if $N \triangleleft G$ then $N \subset \operatorname{Ker} \chi$ and this is not possible as $\rho$ is faithful. Now $[G: H]=2$ and therefore there exists exactly one conjugate of $N$, say $N^{\prime}=\sigma^{-1} N \sigma$. Then $N \cap N^{\prime}=\{i d\}$ because $N \cap N^{\prime} \subset$ $\operatorname{Ker} \chi, N \cap N^{\prime} \triangleleft G$ and $\rho$ is faithful.

Remark 1. If $\mathfrak{K} \neq \mathbb{Q}(\sqrt{-1})$ and $\mathbb{Q}(\sqrt{-3})$, in part (ii) of Lemma 2, we can prove that $n$ is not equal to 8 and 12 . This is true since in these cases $\left.\chi\right|_{H}$ takes values in $\mathbb{Q}\left(\zeta_{8}\right)^{+}$ or $\mathbb{Q}\left(\zeta_{12}\right)^{+}$which are not $\mathbb{Q}$.

Lemma 5 Let $5 \backslash \backslash d_{\mathfrak{K}}$ (discriminant of $\mathfrak{K}$ ). Then, the order of any finite subgroup of $\mathrm{GL}_{2}(\mathcal{O})$ is not divisible by 5 .

Proof: Let $G$ be a finite subgroup of $\mathrm{GL}_{2}(\mathcal{O})$. By Dirichlet's theorem on primes in arithmetic progressions, there are infinitely many primes $q \equiv 2(\bmod 5)$ such that $q$ splits completely in $\mathcal{O}$. Let $q=\mathfrak{q}_{1} \mathfrak{q}_{2}$ in $\mathcal{O}$. We choose $q$ large enough such that the restriction of the reduction map

$$
\mathrm{GL}_{2}(\mathcal{O}) \rightarrow \mathrm{GL}_{2}\left(\mathcal{O} / \mathfrak{q}_{1} \mathcal{O}\right)
$$


to $G$ is injective. But $\operatorname{Card}\left(\mathrm{GL}_{2}\left(\mathcal{O} / \mathfrak{q}_{1} \mathcal{O}\right)\right)=\operatorname{Card}\left(\mathrm{GL}_{2}(\mathbb{Z} / q \mathbb{Z})\right)=\left(q^{2}-1\right)\left(q^{2}-q\right) \equiv 1$ $(\bmod 5)$. This proves the lemma.

Lemma 6 Let $G$ be a subgroup of $\mathrm{GL}_{2}(\mathcal{O})$, then either $G$ is Abelian or $\operatorname{Cent}(G) \simeq$ $\{i d\}, \mathbb{Z} / 2 \mathbb{Z}, \mathbb{Z} / 3 \mathbb{Z}, \mathbb{Z} / 4 \mathbb{Z}, \mathbb{Z} / 6 \mathbb{Z}$.

Proof: We consider $G$ as a subgroup of $\mathrm{GL}_{2}(\mathfrak{K})$. Let

$$
C(G)=\left\{\alpha \in \mathrm{GL}_{2}(\mathfrak{K}): \alpha \gamma=\gamma \alpha \text { for all } \gamma \in G\right\} \text {. }
$$

Then, $G$ is either Abelian or

$$
C(G)=\left\{\left(\begin{array}{cc}
c & 0 \\
0 & c
\end{array}\right): c \in \mathfrak{K}^{*}\right\}
$$

(see [21], p. 179, problem 2.6. (a)). Now the lemma follows from the facts that

$$
\operatorname{Cent}(G)=C(G) \cap G
$$

and $\mathcal{O}^{*} \simeq\{i d\}, \mathbb{Z} / 2 \mathbb{Z}, \mathbb{Z} / 4 \mathbb{Z}, \mathbb{Z} / 6 \mathbb{Z}$

\section{$4 E(F)$ of $\mathbb{Z}$-rank $1,2,3$ or $\mathcal{O}$-rank 1 or 2}

In this section, we assume that $E(F)$ is infinite of either $\mathbb{Z}$-rank $\leq 3$ or $\mathcal{O}$-rank $\leq 2$. We apply the results of the previous section to determine the minimal subfield in the case that $E(F)$ has $\mathbb{Z}$-rank 1,2 or 3 . We also consider the case that $E$ has multiplication by the ring of integers $\mathcal{O}$ of an imaginary quadratic field $\mathfrak{K}$ and $E(F)$ has $\mathcal{O}$-rank 1 or 2. In the latter situation, we are able to determine the minimal subfield in all cases but one.

Theorem 1 Let $E$ be an elliptic curve defined over $K$ and let $F$ be a finite Galois extension of $K$. Let $M$ be the minimal subfield.

(i) If $\operatorname{rank}(E(F))=1$, then $M$ is a cyclic subextension of $K$ and $[M: K]=1$ or 2 .

(ii) If $\operatorname{rank}(E(F))=2$, then $M$ is either a cyclic subextension of $K$ and $[M: K]=$ $1,2,3,4,6$ or a dihedral subextension of $K$ and $[M: K]=4,6,8,12$.

(iii) If $\operatorname{rank}(E(F))=3$, then $\operatorname{Gal}(M / K)$ is one of the following:

$$
C_{n} \times C_{m}, \quad D_{p} \times C_{m}, \quad A_{4} \times C_{m}, \quad S_{4} \times C_{m}
$$

where $n=1,2,3,4,6, p=2,3,4,6$ and $m=1,2$.

Proof: (i) $M / K$ is the subextension given in Proposition 2. It is clear that since $\rho_{f}$ is faithful, $\operatorname{Gal}(M / K)$ is isomorphic to a subgroup of $\mathrm{GL}_{1}(\mathbb{Z}) \simeq \mathbb{Z}^{*}=\{ \pm 1\}$ which is cyclic and has order 1 or 2 . 
(ii), (iii) Let $\rho_{f}$ be the faithful representation given in Proposition 2. Applying Lemmas 1 and 3 on $\rho_{f}$ imply the results.

Now we show that in part (ii) of Theorem 1, $M$ cannot be a dihedral extension of degree 12 of $K$, if we assume the Birch and Swinnerton-Dyer conjecture and some other assumptions.

Let $M$ be a dihedral extension of $\mathbb{Q}$ and let $C$ be the fixed field of the cyclic subgroup $H$ of the dihedral Galois group in $M / \mathbb{Q}$. So $[C: \mathbb{Q}]=2$ and $[M: C]=n$ (say) $(n \geq 3)$. We have

$$
L(E / M, s)=L(E / C, s) \prod_{i} L\left(E / \mathbb{Q} \otimes \operatorname{Ind}_{H}^{G} \psi_{i}, s\right)^{2}
$$

where $\psi_{i}$ are characters of $H=\operatorname{Gal}(M / C)$. Since $G$ is dihedral, the twisted $L$-function $L\left(E / \mathbb{Q} \otimes \operatorname{Ind}_{H}^{G} \psi_{i}, s\right)$ has root number \pm 1 , depending on the parity of the order of vanishing of the twisted $L$-function at $s=1$.

Now assume that the Birch and Swinnerton-Dyer conjecture is true. Then the assumption that $\operatorname{rank}(E(M))=2$, and the above factorization of $L$-functions implies that we have the following possibilities:

(i) $L(E / C, 1)=0$

(ii) exactly one of the factors $L\left(E / \mathbb{Q} \otimes \operatorname{Ind}_{H}^{G} \psi_{i}, s\right)$ has a simple zero at $s=1$. In the first case, we must have $L(E / C, s)$ vanishing to order 2 at $s=1$ and none of the two-dimensional twists vanishes. In particular, all the root numbers must satisfy

$$
w\left(E / \mathbb{Q} \otimes \operatorname{Ind}_{H}^{G} \psi_{i}\right)=1
$$

for all $i$. In the second case, $L(E / C, 1) \neq 0$ and there is a unique $i$ such that $L(E / \mathbb{Q} \otimes$ $\left.\operatorname{Ind}_{H}^{G} \psi_{i}, 1\right)=0$. Since this zero is simple

$$
w\left(E / \mathbb{Q} \otimes \operatorname{Ind}_{H}^{G} \psi_{i}\right)=-1 .
$$

Moreover, as none of the others vanish, all of the other root numbers are equal to +1 .

Now it is clear that if $M$ is the minimal subfield then (i) cannot be true and thus we are in the situation (ii).

Proposition 4 Let $E$ be a modular elliptic curve of conductor $N$ defined over $\mathbb{Q}$ and suppose that the Birch and Swinnerton-Dyer conjecture is true. Also with the above notation assume that $N$ and conductor of $\operatorname{Ind}_{H}^{G} \psi_{i}$ 's are relatively prime and for all $i$, $\chi_{i}=\operatorname{det}\left(\operatorname{Ind}_{H}^{G} \psi_{i}\right)$ is even. Then, in part (ii) of Theorem 1 (for $K=\mathbb{Q}$ ) the minimal subfield $M$ cannot be a dihedral extension of degree 12.

Proof: Let $M$ be the minimal subfield in Theorem 1 and follow the notations before the proposition. By a result of Rohrlich (see [16], p. 125, Proposition 1), the root number can be calculated as follows. Let $\chi_{i}$ be the determinant of $\operatorname{Ind} d_{H}^{G} \psi_{i}$. If $\chi_{i}$ is even, then

$$
w\left(E / \mathbb{Q} \otimes \operatorname{Ind}_{H}^{G} \psi_{i}\right)=\chi_{i}(N) .
$$


Now, $\chi_{i}$ is a quadratic character which can be computed by the following formula:

$$
\chi_{i}=\epsilon \psi_{i} \circ \mathrm{Ver}
$$

where $\epsilon$ is the character of $C / \mathbb{Q}$ and Ver is the transfer map (Verlagerung) given by

$$
\operatorname{Ver}(g)= \begin{cases}g^{2} & \text { if } g \notin H \\ g \cdot \delta g \delta^{-1} & \text { if } g \in H\end{cases}
$$

Here, $\delta$ is a fixed element of $G-H$ of order 2. Now, $\psi\left(\delta g \delta^{-1}\right)=\overline{\psi(g)}$ and so $\psi \circ$ Ver is trivial on $H$. Moreover, $\operatorname{Ver}(\delta)=1$. Hence, $\psi_{i} \circ \operatorname{Ver}=1$ and $\chi_{i}=\epsilon$ is a quadratic character independent of $\psi_{i}$. Thus, the root numbers $w\left(E / \mathbb{Q} \otimes \operatorname{Ind} d_{H}^{G} \psi_{i}\right)$ 's are all equal. But from the argument before the proposition, we know that there is a unique $i$ such that $w\left(E / \mathbb{Q} \otimes \operatorname{Ind}_{H}^{G} \psi_{i}\right)=-1$ and all of the others are +1 . Now since the number of irreducible two dimensional characters of $D_{n}$ is $\frac{n-1}{2}$ if $n$ is odd and $\frac{n-2}{2}$ if $n$ is even, we have $\epsilon(N)=-1$ and $n=3$ or 4 .

Now let $E$ be an elliptic curve defined over a number field $K$ which has complex multiplication by $\mathcal{O}$, the ring of integers of an imaginary quadratic number field $\mathfrak{K}$ contained in $K(\mathfrak{K} \subseteq K)$, and let $F$ be a finite Galois extension of $K$. (We fix once and for all an embedding $\mathfrak{K} \hookrightarrow \mathbb{C}$.) Since $E$ has complex multiplication by $\mathcal{O}$ and $E$ is defined over $K$, we can fix an isomorphism between the ring of endomorphisms of $E$ and $\mathcal{O}$ and equip $E(F)$ with an $\mathcal{O}$ action. (Note that all the endomorphisms of $E$ are defined over $K$.)

We consider the submodule $m E(F)$ of the $\mathcal{O}$-module $E(F)$, where $m$ is the order of the $\mathcal{O}$-torsion submodule of $E(F)$, then $m E(F)$ is a finitely generated torsion free module over $\mathcal{O}$ which is projective since $\mathcal{O}$ is a Dedekind domain. Moreover, there exist free $\mathcal{O}$-modules $M_{1}$ and $M_{2}$, such that

$$
M_{1} \subset m E(F) \subset M_{2}
$$

and $M_{1}$ and $M_{2}$ have the same rank. We call this common rank, the $\mathcal{O}$-rank of $E(F)$. (For the above algebraic facts, see [8], p. 168, Problems 11 and 13.) Note that $2 \operatorname{rank}_{\mathcal{O}}(E(F))=\operatorname{rank}(E(F))$.

Remark 2. If the field of complex multiplication $\mathfrak{K}$ is not contained in $K$, still we can consider $E(F)$ as an $\mathcal{O}$-module if we assume that $\mathfrak{K} K \subset F$. Also, we want to mention that the upcoming results in this section are also valid for elliptic curves with complex multiplication by a non-maximal order in $\mathfrak{K}$.

Now we can consider the $\mathfrak{K}$-module $m E(F) \otimes_{\mathcal{O}} \mathfrak{K}=E(F) \otimes_{\mathcal{O}} \mathfrak{K}$ as a representation space for $\operatorname{Gal}(F / K)$ to get the following representation:

$$
\rho: \operatorname{Gal}(F / K) \rightarrow \operatorname{Aut}\left(E(F) \otimes_{\mathcal{O}} \mathfrak{K}\right) \simeq \mathrm{GL}_{r}(\mathfrak{K})
$$

where $r=\operatorname{rank}_{\mathcal{O}}(E(F))$. It is clear that we can define an $\mathcal{O}$-analogue of the minimal subfield and establish an $\mathcal{O}$-analogue of Propositions 1, 2 and 3. Note that in the 
$\mathcal{O}$-analogue of Proposition 2, we have to assume that $r$ and $h_{\mathfrak{K}}$ (the class number of $\mathfrak{K})$ are relatively prime to make sure that $\operatorname{Im}\left(\rho_{f}\right)$ is conjugate to a finite subgroup of $G L_{r}(\mathcal{O})$. (For more explanation about this condition see [4], Theorem 23.17, p. 530.) Also note that if $\operatorname{rank}_{\mathcal{O}}(E(F))=r$ then the $\mathcal{O}$-minimal subfield is the same as the minimal subfield $F_{2 r}$ defined in the beginning of Section 2.

Proposition 5 If $\operatorname{rank}_{\mathcal{O}}(E(F))=1$, then the minimal subfield is a cyclic subextension $M$ of $K$ and $[M: K]=1,2,3,4$ or 6 .

Proof: Since $\left(h_{\mathfrak{K}}, 1\right)=1$, the argument before the proposition implies that $\operatorname{Im}\left(\rho_{f}\right)$ can be considered as a subgroup of $\mathrm{GL}_{1}(\mathcal{O})$. Now the proof is exactly the $\mathcal{O}$-analogue of part (i) of Theorem 1. Note that $\mathrm{GL}_{1}(\mathcal{O}) \simeq \mathcal{O}^{*}$ which is cyclic and has order 1,2 , 4 or 6 .

If $\operatorname{rank}_{\mathcal{O}}(E(F))=2$ and $h_{\mathfrak{K}}$ is odd, then $\rho(\operatorname{Gal}(F / K))$ is isomorphic to a finite subgroup of $\mathrm{GL}_{2}(\mathcal{O})$. We apply the group theoretic lemmas of the previous section to obtain some useful information about the representation $\rho$ and the group $\operatorname{Gal}(F / K)$.

Theorem 2 Suppose that $h_{\mathfrak{K}}$ is odd and $\operatorname{rank}_{\mathcal{O}}(E(F))=2$. Then there is a Galois subextension $K \subseteq S \subseteq F$ with $\operatorname{rank}_{\mathcal{O}}(E(S))>0$ such that $G=\operatorname{Gal}(S / K)$ is one of the following:

(i) $G$ is cyclic of order $1,2,3,4,6,8$, or 12 .

(ii) $G / \operatorname{Cent}(G) \simeq D_{n}$. More precisely $G$ satisfies one of the following:

(a) $G \simeq D_{3}$.

(b) $\operatorname{Cent}(G) \simeq \mathbb{Z} / 2 \mathbb{Z}$ and $G / \operatorname{Cent}(G) \simeq D_{n}(n=2,3,4,6,8)$.

(c) $\operatorname{Cent}(G) \simeq \mathbb{Z} / 3 \mathbb{Z}$ and $G / \operatorname{Cent}(G) \simeq D_{n}(n=2,3,4,6)$.

(d) $\operatorname{Cent}(G) \simeq \mathbb{Z} / 4 \mathbb{Z}$ and $G / \operatorname{Cent}(G) \simeq D_{n}(n=2,3,4)$.

(e) $\operatorname{Cent}(G) \simeq \mathbb{Z} / 6 \mathbb{Z}$ and $G / \operatorname{Cent}(G) \simeq D_{n}(n=2,3,6)$.

(iii) $\operatorname{Cent}(G) \neq\{$ id $\}$ and $G / \operatorname{Cent}(G) \simeq A_{4}$ or $S_{4}$.

In (ii) and (iii), $\operatorname{rank}_{\mathcal{O}}(E(S))=2$. In fact, $S$ is the minimal subfield in these cases.

Proof: Let $\rho: \operatorname{Gal}(F / K) \rightarrow G L_{2}(\mathcal{O})$ be the representation of $\operatorname{Gal}(F / K)$ in $E(F) \otimes_{\mathcal{O}} \mathfrak{K}$ and $\chi$ be its character. By the $\mathcal{O}$-analogue of Proposition 2, we can assume that $\rho$ is faithful. Also we know that $G / \operatorname{Cent}(G)$ is isomorphic to a finite subgroup of $\mathrm{PGL}_{2}(\mathbb{C})$ and therefore (see [20]) is isomorphic to $C_{n}, D_{n}, A_{4}, S_{4}$ or $A_{5}$. By Lemma $5, G / \operatorname{Cent}(G)$ cannot be isomorphic to $A_{5}$. Note that since $h_{\mathfrak{K}}$ is odd, $\mathfrak{K}=\mathbb{Q}(\sqrt{-p})$ for prime $p$ with $-p \equiv 1(\bmod 4)$ or $\mathfrak{K}=\mathbb{Q}(\sqrt{-1}), \mathbb{Q}(\sqrt{-2})$, and therefore $5 \not d_{\mathfrak{K}}$.

If $\rho$ is reducible, let $\chi$ be the character of $\rho$. We have $\chi=\psi_{1}+\psi_{2}$ over $\mathbb{C}$, where $\psi_{1}$ and $\psi_{2}$ are one dimensional characters of $G$. Let $S$ be the fixed field of $K e r \psi_{1}$ in $F / K$. Then $\psi_{1}$ is a faithful and irreducible character of $\operatorname{Gal}(S / K)$, which implies that $\operatorname{Gal}(S / K)$ is cyclic and $\operatorname{rank}_{\mathcal{O}}(E(S)) \neq 0$. Indeed, (see [16], p. 126)

$$
\left(E(F) \otimes_{\mathcal{O}} \mathbb{C}\right)^{\operatorname{Gal}(F / S)}=E(S) \otimes_{\mathcal{O}} \mathbb{C} .
$$

Now a characteristic polynomial argument similar to the one in Lemma 1 implies that $[S: K]=1,2,3,4,6,8$ or 12 . 
Thus, we may suppose that $\rho$ is irreducible. Then, since $G$ is not Abelian $G / \operatorname{Cent}(G)$ cannot be cyclic. Suppose that $G / \operatorname{Cent}(G)$ is isomorphic to $A_{4}$ or $S_{4}$. In this case, we must have $\operatorname{Cent}(G) \neq\{1\}$. Indeed, $G$ is not isomorphic to $A_{4}$, since $A_{4}$ does not have any 2-dimensional irreducible representation. This also implies that if $G \simeq S_{4}$, and $\chi$ is the character of $\rho$ then $\chi=\operatorname{Ind}_{A_{4}}^{S_{4}} \psi, \psi(1)=1$ (see part (i) of Lemma 4). But it is known that any 1-dimensional representation of $A_{4}$ is trivial on the Klein 4-group $V_{4}$ (see [19], p. 42). Since $V_{4} \triangleleft S_{4}$, we have

$$
V_{4} \subset \operatorname{Ker}\left(\operatorname{Ind}_{A_{4}}^{S_{4}} \psi\right)=\operatorname{Ker} \chi
$$

However, $\chi$ is the character of the faithful representation $\rho$. This is a contradiction. Therefore, $G$ is not isomorphic to $S_{4}$.

It remains to analyze the possibility $G / \operatorname{Cent}(G) \simeq D_{n}$. Let $A$ be the cyclic subgroup of order $n$ in $D_{n}$. Let $L$ be the fixed field of $\operatorname{Cent}(G)$ in $F / K$ and $M$ be the fixed field of $A$ in $L / K$. If $H=\operatorname{Gal}(F / M)$ then $H / \operatorname{Cent}(G) \simeq A$ is cyclic and therefore $H$ is Abelian. Clearly $H$ has index 2 in $G$, thus by part (i) of Lemma $4, \chi=\operatorname{Ind}_{H}^{G} \psi$, $\psi(1)=1$. Let $N=\operatorname{Ker} \psi$.

By part (ii) of Lemma 4 if $N=\{i d\}$, then $H \simeq C_{n}(n=2,3,4,6,8,12)$. By Lemma $6, \operatorname{Cent}(G) \simeq\{i d\}, \mathbb{Z} / 2 \mathbb{Z}, \mathbb{Z} / 3 \mathbb{Z}, \mathbb{Z} / 4 \mathbb{Z}$ or $\mathbb{Z} / 6 \mathbb{Z}$. As $\operatorname{Cent}(G) \subseteq H$, we have the following possibilities. If $\operatorname{Cent}(G) \simeq\{i d\}$ then $G \simeq D_{n}$. In this case $n$ must be odd, since $\operatorname{Cent}\left(D_{n}\right) \neq\{i d\}$ for $n$ even. This proves that $G \simeq D_{3}$. If $\operatorname{Cent}(G) \simeq \mathbb{Z} / 2 \mathbb{Z}$ then $G / \operatorname{Cent}(G) \simeq D_{n}(n=1,2,3,4,6)$. But $n \neq 1$ since in that case $G$ is Abelian. Similarly, if $\operatorname{Cent}(G) \simeq \mathbb{Z} / 3 \mathbb{Z}$ then $G / \operatorname{Cent}(G) \simeq D_{n}(n=2,4)$, if $\operatorname{Cent}(G) \simeq \mathbb{Z} / 4 \mathbb{Z}$ then $G / \operatorname{Cent}(G) \simeq D_{n}(n=2,3)$ and if $\operatorname{Cent}(G) \simeq \mathbb{Z} / 6 \mathbb{Z}$ then $G / \operatorname{Cent}(G) \simeq D_{n}$ $(n=2)$.

Now suppose that $N \neq\{i d\}$. First note that since $\chi=\operatorname{Ind}_{H}^{G} \psi, \psi(1)=1$, then $\left.\chi\right|_{H}=\psi+\psi^{\sigma}$ where $\sigma \in G-H$ and $\psi^{\sigma}(x)=\psi\left(\sigma^{-1} x \sigma\right)$ for $x \in H$ (See [19], Proposition 22, p. 58). This shows that $\operatorname{Ker} \psi^{\sigma}=\sigma^{-1} N \sigma \neq\{i d\}$. Let $R$ be the fixed field of $N$ in $F / M$, since $F$ is the minimal subfield and $K \subset R \subsetneq F$, it is clear that $\operatorname{rank}_{\mathcal{O}}(E(R))=1$. In a similar way, we can show that $\operatorname{rank}_{\mathcal{O}}\left(E\left(R^{\sigma}\right)\right)=1\left(R^{\sigma}\right.$ is the fixed field of $\sigma^{-1} N \sigma$ in $\left.F / M\right)$.

Now since $\operatorname{rank}_{\mathcal{O}}(E(R))=1$, the action of $\operatorname{Gal}(R / M)$ on $E(R) \otimes_{\mathcal{O}} \mathfrak{K}$ is given by $\psi$. This shows that $R$ is the minimal subfield and therefore it is cyclic of degree 1,2 , 3, 4, 6 (Proposition 5). A similar statement holds for $R^{\sigma}$.

By part (iii) of Lemma 4,

$$
\operatorname{Ker} \psi \cap \operatorname{Ker} \psi^{\sigma}=N \cap \sigma^{-1} N \sigma=\{i d\}
$$

This implies that $F=R R^{\sigma}$. Hence,

$$
|H|=[F: M]=\frac{[R: M]\left[R^{\sigma}: M\right]}{\left[R \cap R^{\sigma}: M\right]}=\frac{[R: M]^{2}}{\left[R \cap R^{\sigma}: M\right]} .
$$


An easy calculation implies that $[F: M]=4,8,9,12,16,18,36$, which can be checked from the following table:

Table 1

\begin{tabular}{c|c|c}
{$[R: M]$} & {$\left[R \cap R^{\sigma}: M\right]$} & {$[F: M]$} \\
\hline 2 & 1 & 4 \\
3 & 1 & 9 \\
4 & 1,2 & 8,16 \\
6 & $1,2,3$ & $12,18,36$.
\end{tabular}

Note that $[R: M] \neq 1$, since otherwise $R=R^{\sigma}=M$.

By Lemma $6, \operatorname{Cent}(G) \simeq\{i d\}, \mathbb{Z} / 2 \mathbb{Z}, \mathbb{Z} / 3 \mathbb{Z}, \mathbb{Z} / 4 \mathbb{Z}$ or $\mathbb{Z} / 6 \mathbb{Z}$. If $|\operatorname{Cent}(G)|=1$ then $G \simeq D_{n}$, this implies that $N \triangleleft G$ and therefore $N \subset K e r \chi$ which is a contradiction since $N \neq\{i d\}$ and $\chi$ is faithful. If $|\operatorname{Cent}(G)|=4$ and $N \neq\{i d\}$, then the proof of Lemma 6 shows that $\mathfrak{K}=\mathbb{Q}(\sqrt{-1})$ and therefore $[R: M]=2$, 4, thus $[F: M]=8,16$ and so $G / \operatorname{Cent}(G) \simeq D_{n}(n=2,4)$. If $|\operatorname{Cent}(G)|=6$ and $N \neq\{i d\}$, then $[F: M]=$ $12,18,36$ and so $G / \operatorname{Cent}(G) \simeq D_{n}(n=4,6,12)$.

If $|\operatorname{Cent}(G)|=2$, we can refine the above argument to show that $[F: M]$ cannot be 9,18 or 36 . Since $H=\operatorname{Gal}(F / M)$ contains $\operatorname{Cent}(G)$, the order of $H$ is even and so $[F: M] \neq 9$. To show that $[F: M] \neq 18$ or 36 , recall that $N \neq\{i d\}$ and $|\operatorname{Cent}(G)|=2$. We first claim that $N$ is a 2 -group (in fact, it is a cyclic ${ }^{1} 2$-group). This is true, because as $N$ and $H$ are Abelian, they can be written as a direct sum of their Sylow subgroups

$$
N=N_{2} \oplus N_{\text {odd }}, \quad H=H_{2} \oplus H_{o d d}
$$

where $\mathrm{N}_{2}$ (respectively $\mathrm{H}_{2}$ ) is the 2-primary part of $N$ (respectively $H$ ). Since $H / C e n t(G)$ is cyclic and $|\operatorname{Cent}(G)|=2$, it follows that $H_{\text {odd }}$ is cyclic. Moreover, $H_{o d d} \triangleleft G$, and since $N_{o d d} \subset H_{o d d}$ and $H_{o d d}$ is cyclic, $N_{o d d} \triangleleft G$. This shows that for $\sigma \in G-H$

$$
N_{\text {odd }} \subset N \cap \sigma^{-1} N \sigma=\{i d\}
$$

and therefore $N=N_{2}$.

Now let $M_{2}$ be the fixed field of $H_{2}$ in $F / M$. Since $N$ is a subgroup of $H_{2}$, it is clear that $R$ (the fixed field of $N$ in $F / M$ ) is a Galois extension of $M_{2}$, and since $R / M$ is cyclic with $[R: M]=1,2,3,4,6, R$ is a cyclic extension of $M_{2}$ and $\left[R: M_{2}\right]=1,2,4$. A similar statement holds for $R^{\sigma} / M_{2}$. We have

$$
|H|=\left[F: M_{2}\right]\left[M_{2}: M\right]=\frac{\left[R: M_{2}\right]^{2}}{\left[R \cap R^{\sigma}: M_{2}\right]}\left[M_{2}: M\right] .
$$

The following table summarizes the possibilities for $[F: M]$ in this case.

\footnotetext{
${ }^{1}$ Note that $N \cap \operatorname{Cent}(G)=\{i d\}$ and $N \simeq N / N \cap \operatorname{Cent}(G) \simeq N \operatorname{Cent}(G) / \operatorname{Cent}(G) \subset H / \operatorname{Cent}(G) \simeq$ $A$, where $A$ is the cyclic subgroup of order $n$ in $D_{n}$.
} 
Table 2

\begin{tabular}{c|c|c|c}
{$\left[R: M_{2}\right]$} & {$\left[R \cap R^{\sigma}: M_{2}\right]$} & {$\left[M_{2}: M\right]$} & {$[F: M]$} \\
\hline 2 & 1 & 1,3 & 4,12 \\
4 & 1,2 & 1 & $8,16$.
\end{tabular}

So if $|\operatorname{Cent}(G)|=2$ and $N \neq\{i d\}$, then $[F: M]=4,8,12,16$ and so $G / \operatorname{Cent}(G) \simeq D_{n}(n=2,4,6,8)$. Similarly, if $|\operatorname{Cent}(G)|=3$ and $N \neq\{i d\}$, we can prove that $N=N_{\text {odd }}$, and $[F: M]=9,18$ and so $G / \operatorname{Cent}(G) \simeq D_{n}(n=3,6)$.

Now it is easy to verify the list given in part (ii) of the statement of the theorem. This completes the proof.

Remark 3. It might be of interest to note that a group $G$ with cyclic center $\operatorname{Cent}(G)$ having the property that $G / \operatorname{Cent}(G) \simeq D_{n}$ is necessarily a product $H K$ with $H$ and $K$ Abelian, with $H \cap K=\operatorname{Cent}(G)$. Moreover, if $\operatorname{Cent}(G)$ has order $m$, then $H$ has order $m n$ and $K$ has order $2 m$. In some cases, we can say more. For example, if $n=3$ and $m=2,3,4$, then $G \simeq \operatorname{Cent}(G) \times D_{n}$.

Definition: The generalized quaternion group $Q_{4 n}$ is defined with the following presentations:

$$
Q_{4 n}=\left\langle x, y: x^{2 n}=1, x^{n}=y^{2}, y x y^{-1}=x^{-1}\right\rangle
$$

Theorem 3 Suppose that $h_{\mathfrak{K}}$ is odd and $\operatorname{rank}_{\mathcal{O}}(E(F))=2$ and $\mathfrak{K} \neq \mathbb{Q}(\sqrt{-1}), \mathbb{Q}(\sqrt{-3})$. Then there is a Galois subextension $S$ with $K \subseteq S \subseteq F$ and $\operatorname{rank}_{\mathcal{O}}(E(S))>0$ such that $G=\operatorname{Gal}(S / K)$ is one of the following:

(i) $G$ is cyclic of order $1,2,3,4$ or 6 .

(ii) $G$ is isomorphic to $D_{n}(n=3,4,6)$ or $Q_{4 n}(n=2,3)$.

(iii) $G \simeq \mathrm{SL}_{2}(\mathbb{Z} / 3 \mathbb{Z})$ or $G$ is isomorphic to an extension of $\mathrm{SL}_{2}(\mathbb{Z} / 3 \mathbb{Z})$ by $\mathbb{Z} / 2 \mathbb{Z}$ with $\operatorname{Cent}(G) \simeq \mathbb{Z} / 2 \mathbb{Z}$. This can occur only if $d_{\mathfrak{K}} \not \equiv 1(\bmod 8)$.

In (ii) and (iii), $\operatorname{rank}_{\mathcal{O}}(E(S))=2$. In fact, $S$ is the minimal subfield in these cases.

Proof: First note that since $\mathfrak{K} \neq \mathbb{Q}(\sqrt{-1}), \mathbb{Q}(\sqrt{-3})$ in part (ii) of Lemma $4, n \neq$ 8, 12 (see Remark 1). Applying this fact in the proof of Theorem 2 implies (i) if $\rho$ (defined in the proof of Theorem 2) is reducible. In the case that $\rho$ is irreducible and $G / \operatorname{Cent}(G) \simeq D_{n}$, from the assumptions of the theorem, we conclude that $G \simeq D_{3}$ or $\operatorname{Cent}(G) \simeq \mathbb{Z} / 2 \mathbb{Z}$ and $G / \operatorname{Cent}(G) \simeq D_{n}(n=2,3)^{2}$. Now it is easy to verify the list given in part (ii) of the statement of the theorem, by referring to the list of non-Abelian groups of order 8 and 12 (see for example [5], Appendix B, p. 238).

So, we may suppose that $\rho$ is irreducible and $G / \operatorname{Cent}(G)$ is isomorphic to either $A_{4}$ or $S_{4}$ and that $\operatorname{Cent}(G) \simeq \mathbb{Z} / 2 \mathbb{Z}$.

\footnotetext{
${ }^{2}$ Note that $\operatorname{Cent}(G) \simeq \mathbb{Z} / 2 \mathbb{Z}$, however, $n=4,6,8$ never occur. This is true since $\mathfrak{K} \neq$ $\mathbb{Q}(\sqrt{-1}), \mathbb{Q}(\sqrt{-3})$ and therefore in the proof of Theorem 2 if $N=\{i d\}$, then $H \simeq C_{n}(n=2,3,4,6)$ and if $N \neq\{i d\}$, then in Table $1,[R: M]=2$.
} 
Let $G / \operatorname{Cent}(G) \simeq A_{4}$. Suppose that $L$ is the fixed field of $C e n t(G)$ in $F / K$ and $M$ is the fixed field of $V_{4}$ (Klein's 4-group) in $L / K$. Set $H \simeq \operatorname{Gal}(F / M)$. Since $H / \operatorname{Cent}(G) \cong V_{4}$ and $V_{4} \triangleleft A_{4}$, it follows that $H \triangleleft G$, also it is clear that $[G: H]=3$. Suppose that $\left.\chi\right|_{H}$ is reducible. Then, by part (i) of Lemma $4, \chi=\operatorname{Ind}_{H}^{G} \psi, \psi(1)=1$. This can never happen because $[G: H]=3$ and $\chi$ is 2 dimensional.

Thus $\left.\chi\right|_{H}$ is irreducible. Note that $H$ is the 2-Sylow subgroup of $G$ and it is of order 8. As it is necessarily non-Abelian, it is isomorphic to either $D_{4}$ or $Q_{8}$ (the quaternion group of order 8$)$. In either case, $G$ is the semidirect product of $H$ and $\mathbb{Z} / 3 \mathbb{Z}$.

If $H \simeq Q_{8}$, then $G \simeq \mathrm{SL}_{2}(\mathbb{Z} / 3 \mathbb{Z})$. This group has three 2-dimensional irreducible representations. For two of these, the character takes values in $\mathbb{Q}(\sqrt{-3})$ (see for example [12], p. 61) and hence we can exclude these. The remaining representation has character values in $\mathbb{Z}$. If the restriction of this representation to $Q_{8}$ is irreducible (as we are assuming), it is a representation of Schur index 2 (see [19], p. 94, Exercise 12.3) and it is realizable over $\mathfrak{K}$ if and only if $\mathfrak{K}$ can be embedded in the quaternion algebra $\mathbb{D}$ over $\mathbb{Q}$ which is ramified at 2 and $\infty$. But if $d_{\mathfrak{K}} \equiv 1(\bmod 8)$, then $\mathfrak{K}$ cannot be embedded in $\mathbb{D}$ as the prime 2 splits in this field. Thus, if $d_{\mathfrak{K}} \equiv 1(\bmod 8)$ this case cannot occur.

If $H \simeq D_{4}$, then let $J$ be the cyclic subgroup of order 4 . Let $A$ be a 3-Sylow subgroup of $G$. It acts by conjugation on $J$ (as $J$ contains all elements of order 4 in $\left.D_{4}\right)$. Moreover, it must act trivially as $\operatorname{Aut}(J)$ is cyclic of order 2 . Hence, $A J$ is cyclic of order 12. Let $P$ be the quadratic extension of $K$ which is fixed by $A J$. Restricting our representation $\rho$ to $A J$, we find it is reducible and given by two characters $\psi_{1}$ and $\psi_{2}$ (say). $\psi_{1}$ and $\psi_{2}$ take values in the group of 12 -th roots of unity. The character of $\rho$ on $H$ thus takes values in $\mathbb{Q}\left(\zeta_{12}\right) \cap \mathfrak{K}=\mathbb{Q}($ as $\mathfrak{K} \neq \mathbb{Q}(\sqrt{-1}), \mathbb{Q}(\sqrt{-3}))$. In particular, it is real and so either $\psi_{1}$ and $\psi_{2}$ are both real or $\psi_{2}$ is the complex conjugate of $\psi_{1}$. Since $\left.\rho\right|_{H}$ is faithful, the first case cannot occur as it would imply that $H$ has order at most 4 . Hence, we are in the second case, and this implies that $\psi_{1}$ is of order 12 . But then, the character takes values in $\mathbb{Q}\left(\zeta_{12}\right)^{+}$which is not $\mathbb{Q}$ and this is a contradiction. Thus, this case also cannot occur.

Let $G / \operatorname{Cent}(G) \simeq S_{4}$. Again let $L$ be the fixed field of $\operatorname{Cent}(G)$ in $F / K, M$ be the fixed field of $A_{4}$ in $L / K$ and $H=\operatorname{Gal}(F / M)$. Suppose first that $\left.\chi\right|_{H}$ is reducible. Then by part (i) of Lemma $4, \chi=\operatorname{Ind}_{H}^{G} \psi, \psi(1)=1$. Let $N=\operatorname{Ker} \psi$. It is clear that $N \neq\{i d\}$, since otherwise by part (ii) of Lemma $4, H$ is cyclic which is impossible. Let $R$ be the fixed field of $N$, then $\operatorname{rank}_{\mathcal{O}}(E(R))>0$. Since $\rho$ is faithful, we must have $\operatorname{rank}_{\mathcal{O}}(E(R))=1$. This implies that $R$ is the minimal subfield and therefore it is cyclic of order 1 or 2 (Proposition 5). Since $N \cap \sigma^{-1} N \sigma=\{i d\}$, we have $F=R R^{\sigma}$ and then by a calculation similar to one used in the proof of Theorem 2 , we deduce $[F: M]=4$ and hence $[F: K]=8$, contradicting our assumption that $G / \operatorname{Cent}(G) \simeq S_{4}$.

Now consider the case $\chi_{1}=\left.\chi\right|_{H}$ is irreducible. We argue as in the $A_{4}$ case. Let us set $H_{1}$ to be the 2-Sylow subgroup of $H$. Note that it is a normal subgroup. Now, if we have $\left.\chi_{1}\right|_{H_{1}}$ reducible, this would force $\rho_{1}$ to be the induction of a character from 
$H_{1}$ to $H$ (by part (i) of Lemma 4) contradicting the fact that $\rho_{1}$ is a 2-dimensional representation. On the other hand, if $\left.\chi_{1}\right|_{H_{1}}$ is irreducible, then $H_{1}$ is either the quaternion group of order 8 or the dihedral group of order 8 and both of these cases are dealt with as in the $A_{4}$ case using the fact that our representation has to be realizable over $\mathfrak{K}$. This shows that if $d_{\mathfrak{K}} \not \equiv 1(\bmod 8)$, then $H \simeq \mathrm{SL}_{2}(\mathbb{Z} / 3 \mathbb{Z})$ and therefore $G$ is an extension of $\mathrm{SL}_{2}(\mathbb{Z} / 3 \mathbb{Z})$ by $\mathbb{Z} / 2 \mathbb{Z}$. This completes the proof of the theorem.

\section{$5 \quad$ Vanishing of $L$-functions}

\section{$5.1 \quad$ Non-CM case}

Let $E$ be an elliptic curve defined over $\mathbb{Q}$ and let $L(E / \mathbb{Q}, s)$ be the $L$-function of $E$ over $\mathbb{Q}$. Kolyvagin $[7]$ proved that for a (modular) elliptic curve $E$ if $\operatorname{rank}(E(\mathbb{Q})) \geq 1$ then $L(E / \mathbb{Q}, 1)=0$ (see $[6]$, p. 356, Theorem 20.5.2. (b)). This result is generalized to any finite Abelian extension of $\mathbb{Q}$ by Kato (unpublished).

Theorem 4 Let $E$ be a modular elliptic curve defined over $\mathbb{Q}$ and let $F$ be a finite solvable extension of $\mathbb{Q}$. Suppose that $\operatorname{rank}(E(F)) \geq 1$.

(i) If $E(F) \otimes \mathbb{Q}$ is an Abelian $\operatorname{Gal}(F / \mathbb{Q})$ module then $L(E / F, 1)=0$.

(ii) If $\operatorname{rank}(E(F))=1$ then $L(E / F, 1)=0$.

(iii) If $\operatorname{rank}(E(F))=2$ then either $L(E / F, 1)=0$ or the minimal subfield is a dihedral extension of $\mathbb{Q}$ of degree 6,8 or 12 .

(iv) If $\operatorname{rank}(E(F))=3$ then either $L(E / F, 1)=0$ or $\operatorname{Gal}(M / K)$ ( $M$ is the minimal subfield) is one of the following:

$$
A_{4}, \quad S_{4}, \quad A_{4} \times C_{2}, \quad S_{4} \times C_{2} .
$$

Proof: (i) Since $E(F) \otimes \mathbb{Q}$ is an Abelian Galois module, by Proposition 2, there is an Abelian subextension $M$ of $\mathbb{Q}$ such that $\operatorname{rank}(E(M)) \geq 1$. Now Kato's generalization of Kolyvagin's theorem implies the vanishing of $L(E / M, s)$ at $s=1$. By Theorem 2 of [11], $L(E / F, s)$ is divisible by $L(E / M, s)$. Hence, $L(E / F, s)$ also vanishes at $s=1$. This completes the proof.

(ii) By part (i) of Theorem $1, E(F) \otimes \mathbb{Q}$ is a cyclic Galois module, and the result follows from part (i).

(iii) It follows from part (ii) of Theorem 1 and (i).

(iv) Let $\rho_{f}: \operatorname{Gal}(M / K) \rightarrow \mathrm{GL}_{3}(\mathbb{Z})$ be the faithful representation given in Proposition 2. We prove that if $\rho_{f}$ is reducible then $L(E / F, 1)=0$. Let $\rho_{f}$ be reducible, then since its degree is $3, \rho_{f}$ has a one dimensional representation $\psi$ of $\operatorname{Gal}(M / K)$ as a direct summand. Let $M_{1}$ be the fixed field of $k e r \psi$ in $M / K$. It is clear that $E$ has a point of infinite order on $M_{1}$ and $M_{1}$ is at most quadratic over $\mathbb{Q}$. As in (i), we conclude that $L\left(E / M_{1}\right)=0$ which implies $L(E / F, 1)=0$. 
Now note that in part (iii) of Theorem 1, the only groups with a possible three dimensional irreducible representation, are those given in the statement of the theorem. This completes the proof.

Remark 4. If $M / \mathbb{Q}$ is a dihedral extension of degree $2 n$ such that the fixed field $C$ of the cyclic subgroup of order $n$ of $\operatorname{Gal}(M / \mathbb{Q})$ is imaginary quadratic and of discriminant prime to the conductor of $E$, and $(E(M) \otimes \mathbb{C})^{\chi} \neq 0$ is infinite ( $\chi$ is a two dimensional character of $\operatorname{Gal}(M / \mathbb{Q}))$, then by recent work of Bertolini and Darmon $[2], L(E / \mathbb{Q} \otimes$ $\chi, 1)=0$. Applying this with the factorization of the $L$-function of $E$ over $M$ (see the paragraph before Proposition 4) and part (ii) of Theorem 1, we deduce that if $F$ is a finite solvable extension of $\mathbb{Q}$ such that any quadratic subfield is imaginary and of discriminant prime to the conductor of $E$, and $\operatorname{rank}(E(F))=2$ then $L(E / F, 1)=0$.

\subsection{CM case}

Let $E$ be an elliptic curve defined over an imaginary quadratic field $K$ and having complex multiplication by $\mathcal{O}$, the ring of integers of $K$. Let $L(E / K, s)$ be the $L$-function of $E$ over $K$. It is known that $L(E / K, s)$ is the product of two Hecke $L$-series of $K$ (see [21], p. 175, Theorem 10.5) and therefore it is defined on the whole complex plane. Coates and Wiles [3] proved that if $\operatorname{rank}(E(K)) \geq 1$ then $L(E / K, 1)=0$. Arthaud [1] generalized this result to any finite Abelian extension of $K$. She proved that if $F$ is a finite Abelian extension of $K \operatorname{such}$ that $\operatorname{rank}(E(F)) \geq 1$ then $L(E / F, 1)=0$. The work of Rubin [18] established this under some conditions even if $E$ is not defined over $K$.

Theorem 5 Let $E$ be an elliptic curve defined over an imaginary quadratic field $K$ and having complex multiplication by $\mathcal{O}$, the ring of integers of $K$. Let $F / K$ be a finite Galois extension and let $\operatorname{rank}_{\mathcal{O}}(E(F)) \geq 1$.

(i) If $E(F) \otimes_{\mathcal{O}} K$ is an Abelian $K[G]$-module then $L(E / F, 1)=0$.

(ii) If $\operatorname{rank}_{\mathcal{O}}(E(F))=1$ then $L(E / F, 1)=0$.

(iii) If $\operatorname{rank}_{\mathcal{O}}(E(F))=2$ and $K \neq \mathbb{Q}(\sqrt{-1}), \mathbb{Q}(\sqrt{-3})$, then either $L(E / F, 1)=0$ or the Galois group of the minimal subfield over $K$ is isomorphic to one of the following:

a) $D_{n}(n=3,4,6), Q_{4 n}(n=2,3)$.

b) $\mathrm{SL}_{2}(\mathbb{Z} / 3 \mathbb{Z})$ or an extension of $\mathrm{SL}_{2}(\mathbb{Z} / 2 \mathbb{Z})$ by $\mathbb{Z} / 2 \mathbb{Z}$ with $C$ ent $(G) \simeq \mathbb{Z} / 2 \mathbb{Z}$. This can occur only if $K \neq \mathbb{Q}(\sqrt{-7})$.

Proof: (i) By the $\mathcal{O}$-analogue of Proposition 2, there is an Abelian subextension $M$ of $K$ such that $\operatorname{rank}_{\mathcal{O}}(E(M)) \geq 1$. Now by Arthaud's theorem [1], $L(E / M, 1)=0$. By Theorem 1 of $[11], L(E / F, s)$ is divisible by $L(E / M, s)$. Hence $L(E / F, 1)=0$.

(ii) By Proposition $5, E(F) \otimes_{\mathcal{O}} K$ is a cyclic $K[G]$-module, and the result follows from part (i).

(iii) It follows from Theorem 3 and (i). Note that since the $j$-invariant $j(E) \in K$ then $h_{K}=1$, and $K=\mathbb{Q}(\sqrt{-7})$ is the only imaginary quadratic number field with $h_{K}=1$ that for it $d_{K} \equiv 1(\bmod 8)$. 


\section{Elliptic analogue of Stark's theorem}

In this section, we investigate the analytic analogue of the minimal subfield. In this, we are guided by the results of Stark [22] about simple zeros of Dedekind zeta functions.

Definition: Let $E$ be an elliptic curve defined over $K$ and let $F$ be an extension of $K$. For each zero $\omega$ of $L(E / F, s)$, the analytic minimal subfield $F_{\omega}$ is a subfield over $K$ with $K \subseteq F_{\omega} \subseteq F$ such that

(i) $\operatorname{ord}_{s=\omega} L\left(E / F_{\omega}, s\right)=\operatorname{ord}_{s=\omega} L(E / F, s)$.

(ii) If $K \subseteq M \subseteq F$ and $\operatorname{ord}_{s=\omega} L(E / M, s)=\operatorname{ord}_{s=\omega} L(E / F, s)$, then $F_{\omega} \subseteq M$.

Proposition 6 Let $F / K$ be a Galois extension with Galois group $G$, and suppose that $L(E / K \otimes \chi, s)$ is holomorphic at $s=\omega$ for any irreducible character $\chi$ of $G$. Then the analytic minimal subfield $F_{\omega}$ exists and it is Galois over $K$.

Proof: We have the factorization

$$
L(E / F, s)=\prod_{\chi \in \operatorname{Irr}(G)} L(E / K \otimes \chi, s)^{\chi(1)}
$$

where $\operatorname{Irr}(G)$ is the set of irreducible characters of $G$. Consider the set

$$
Z_{\omega}=\{\chi \mid L(E / K \otimes \chi, \omega)=0\}
$$

Define

$$
H_{\omega}=\bigcap_{\chi \in Z_{\omega}} \operatorname{Ker} \chi
$$

Then $H_{\omega}$ is a normal subgroup of $G$ and we let $F_{\omega}$ denote its fixed field, which is Galois over $K$. Using the holomorphy of $L(E / K \otimes \chi, s)$, it is easy to see that $\operatorname{ord}_{s=\omega} L(E / F, s)=\operatorname{ord}_{s=\omega} L\left(E / F_{\omega}, s\right)$.

Now let $M$ be any field between $F$ and $K$. Put $H=\operatorname{Gal}(F / M)$ and let $1_{H}$ be the identity character of $H$. We have

$$
\operatorname{Ind}_{H}^{G} 1_{H}=\sum_{\chi \in \operatorname{Irr}(G)} a_{\chi} \chi, \quad 0 \leq a_{\chi} \leq \chi(1), \quad a_{\chi} \in \mathbb{Z} .
$$

Thus,

$$
L(E / M, s)=L\left(E / K \otimes I n d{ }_{H}^{G} 1_{H}, s\right)=\prod_{\chi \in \operatorname{Irr}(G)} L(E / K \otimes \chi, s)^{a_{\chi}} .
$$

This shows that if $\operatorname{ord}_{s=\omega} L(E / M, s)=\operatorname{ord}_{s=\omega} L(E / F, s)$, then

$$
\sum a_{\chi} n_{\chi}=\sum \chi(1) n_{\chi}
$$


where $n_{\chi}$ denotes the order of $L(E / K \otimes \chi, s)$ at $s=\omega$. Hence, $a_{\chi}=\chi(1)$ for all $\chi \in Z_{\omega}$. We have

$$
a_{\chi}=\left\langle\operatorname{Ind}{ }_{H}^{G} 1_{H}, \chi\right\rangle_{G}=\left\langle 1_{H},\left.\chi\right|_{H}\right\rangle_{H}=\frac{1}{|H|} \sum_{g \in H} \chi(g) .
$$

Now if $a_{\chi}=\chi(1)$, then as $|\chi(g)| \leq \chi(1)$, we must have $\chi(g)=\chi(1)$ for all $g \in H$ and therefore $H \subset \operatorname{Ker} \chi$ and this holds for all $\chi \in Z_{\omega}$. In other words $H \subset H_{\omega}$. This proves that $F_{\omega} \subseteq M$.

Definition. We say that $E$ satisfies the Taniyama conjecture over a field $K$ if the $L$-function $L(E / K, s)$ is the $L$-function $L(\pi, s)$ of an automorphic representation of $\mathrm{GL}_{2}\left(\mathbb{A}_{K}\right)$, where $\mathbb{A}_{K}$ is the adèle ring of $K$.

Proposition 7 Suppose that $E$ satisfies the Taniyama conjecture over $K$. Let $F$ be a solvable extension of $K$ and let $\chi$ be a character of $G=\operatorname{Gal}(F / K)$. Then, $L(E / K \otimes$ $\chi, s)$ is holomorphic at $s=\omega$ if $\omega$ is a simple zero of $L(E / F, s)$.

Proof: Let $H$ be a subgroup of $G$ and let $\chi$ and $\psi$ denote irreducible characters of $G$ and $H$. Set

$$
\theta_{G}=\sum_{\chi} n_{\chi} \chi, \quad \theta_{H}=\sum_{\psi} n_{\psi} \psi
$$

where $n_{\chi}$ and $n_{\psi}$ denote the orders of zeros of $L(E / K \otimes \chi, s)$ and $L\left(E / F^{H} \otimes \psi, s\right)$ at $s=\omega$ respectively $\left(F^{H}\right.$ is the fixed field of $H$ in $\left.F / K\right)$. By Proposition 1 of [11]

$$
\left.\theta_{G}\right|_{H}=\theta_{H}
$$

Suppose $g$ is an element of $G$ and let $H=\langle g\rangle$ be the cyclic group generated by $g$. Then, $L\left(E / F^{H} \otimes \psi, s\right)$ is analytic (see [11], p. 492, Proof of Theorem 2) and since

$$
L(E / F, s)=\prod_{\psi} L\left(E / F^{H} \otimes \psi, s\right)^{\psi(1)}
$$

and $\operatorname{ord}_{s=\omega} L(E / F, s)=1$, then $\theta_{H}=\psi$ for some irreducible character $\psi$ of $H$. From $(*), \theta_{G}(g)$ is a root of unity and therefore

$$
\begin{gathered}
\sum_{\chi} n_{\chi}{ }^{2}=\left\langle\sum_{\chi} n_{\chi} \chi, \sum_{\chi} n_{\chi} \chi\right\rangle \\
=\frac{1}{|G|} \sum_{g \in G}\left|\theta_{G}(g)\right|^{2}=1 .
\end{gathered}
$$

This shows that all $n_{\chi}$ 's except one are 0 . By taking $H=\langle 1\rangle$, we have $\theta_{G}(1)=1$ and thus the remaining $n_{\chi}$ is 1 . This proves that $L(E / K \otimes \chi, s)$ is analytic at $s=\omega$. 
Corollary 1 Under the assumptions of the above proposition $F_{\omega}$ exists. Moreover, $F_{\omega}$ is a cyclic extension of $K$. If $\omega$ is real, $\left[F_{\omega}: K\right] \leq 2$.

Proof: By the previous proposition $L(E / K \otimes \chi, s)$ is holomorphic at $s=\omega$, thus if $\operatorname{ord}_{s=\omega} L(E / F, s)=1$ then there is a $\chi \in \operatorname{Irr}(G)$ such that $\operatorname{ord}_{s=\omega} L(E / K \otimes \chi, s)=1$ and $\chi(1)=1$. Now by Proposition $6, F_{\omega}$ is the fixed field of $\operatorname{Ker} \chi$. Since $\chi$ is one dimensional $F_{\omega}$ is a cyclic extension of $K$. Moreover, if $\omega$ is real

$$
\operatorname{ord}_{s=\omega} L(E / K \otimes \bar{\chi}, s)=\operatorname{ord}_{s=\omega} L(E / K \otimes \chi, s) .
$$

Hence, $\chi=\bar{\chi}$.

Remark 5. Let $F$ be a Galois extension of $K$, then Corollary 1 is still true if $E$ is an elliptic curve with complex multiplication. Note that in this case, we can remove the hypothesis that $F / K$ is solvable, as $E$ satisfies the Taniyama conjecture over any Galois extension of $K$ (see [11], p. 488, Lemma 2).

Corollary 2 Let $E$ be an elliptic curve defined over a number field $K$. Suppose that $E$ has complex multiplication by an order in an imaginary quadratic field contained in $K$. Let $F$ be a Galois extension of $K$ and let $\chi$ be a character of $G=\operatorname{Gal}(F / K)$. Then, $L(E / K \otimes \chi, s)$ is holomorphic at $s=\omega$ if $\omega$ is a double zero of $L(E / F, s)$, and $\omega$ is real. Moreover, $F_{\omega}$ exists and $F_{\omega}$ is a cyclic extension of $K$.

Proof: We have the factorization

$$
L(E / K, s)=L\left(\psi_{K}, s\right) L\left(\bar{\psi}_{K}, s\right)
$$

where $\psi_{K}$ is a Hecke character of $K$. Over $F$,

$$
L(E / F, s)=L\left(\psi_{F}, s\right) L\left(\overline{\psi_{F}}, s\right)
$$

where $\psi_{F}$ denotes the restriction of $\psi_{K}$ to $\operatorname{Gal}(\bar{F} / F)$. As $\omega$ is real, both factors on the right vanish at $s=\omega$. As $\operatorname{ord}_{s=\omega} L(E / F, s)=2$, it follows that

$$
\operatorname{ord}_{s=\omega} L\left(\psi_{F}, s\right)=\operatorname{ord}_{s=\omega} L\left(\overline{\psi_{F}}, s\right)=1 .
$$

Now the argument of Proposition 7 implies that all $L\left(\psi_{K} \otimes \chi, s\right)$ are holomorphic at $s=\omega$ and that $F_{\omega}$ exists and is a cyclic extension of $K$.

Finally, we show that we can replace the assumption of holomorphy in the statement of Proposition 6, with a milder condition if we assume that $E$ has complex multiplication and $F$ is contained in a solvable extension of $K(F / K$ is not necessarily Galois).

Proposition 8 Suppose that $F / K$ has solvable normal closure, and let $E$ be an elliptic curve defined over $K$ which has complex multiplication. Suppose that for any two subfields $M_{1}$ and $M_{2}$ with the property that

$$
\operatorname{ord}_{s=\omega} L\left(E / M_{1}, s\right)=\operatorname{ord}_{s=\omega} L\left(E / M_{2}, s\right)=\operatorname{ord}_{s=\omega} L(E / F, s)
$$


the quotient

$$
\frac{L\left(E / M_{1} M_{2}, s\right) L\left(E / M_{1} \cap M_{2}, s\right)}{L\left(E / M_{1}, s\right) L\left(E / M_{2}, s\right)}
$$

is holomorphic at $s=\omega$. Then the analytic minimal subfield $F_{\omega}$ exists.

Proof: Let $\mathcal{S}$ be the set of subfields $M$ of $F$ with

$$
\operatorname{ord}_{s=\omega} L(E / M, s)=\operatorname{ord}_{s=\omega} L(E / F, s) .
$$

We prove that $\mathcal{S}$ is closed under intersections and thus has a minimal element. Let $M_{1}$ and $M_{2}$ be in $\mathcal{S}$, then by the hypothesis

$$
\frac{L\left(E / M_{1} M_{2}, s\right) L\left(E / M_{1} \cap M_{2}, s\right)}{L\left(E / M_{1}, s\right) L\left(E / M_{2}, s\right)}
$$

is holomorphic at $\omega$. Moreover, by the main result of [11] (see Theorem 1), $L\left(E / M_{1}, s\right)$ divides $L\left(E / M_{1} M_{2}, s\right)$ and $L\left(E / M_{1} M_{2}, s\right)$ divides $L(E / F, s)$. Thus,

$$
\operatorname{ord}_{s=\omega} L\left(E / M_{1}, s\right) \leq \operatorname{ord}_{s=\omega} L\left(E / M_{1} M_{2}, s\right) \leq \operatorname{ord}_{s=\omega} L(E / F, s)
$$

and therefore we have equality throughout. Hence,

$$
\operatorname{ord}_{s=\omega} L\left(E / M_{1} \cap M_{2}, s\right) \geq \operatorname{ord}_{s=\omega} L(E / F, s) .
$$

The reverse inequality also holds (as $L\left(E / M_{1} \cap M_{2}, s\right)$ divides $L(E / F, s)$ ). This proves that $\mathcal{S}$ has a minimal element $F_{\omega}$.

Remark 6. Note that the assumption of holomorphy in the previous proposition is implied by the holomorphy of $L(E / K \otimes \chi, s)$ at $s=\omega$ (see [22], p. 151, Lemma 12).

Remark 7. Proposition 8 is also true, in the case that $E$ satisfies the Taniyama conjecture over $K$ and $F$ is a solvable extension of $K$.

\section{References}

[1] N. ARthaud, On Birch and Swinnerton-Dyer's conjecture for elliptic curves with complex multiplication. I., Comp. Math. 37 (1978), 209-232.

[2] M. Bertolini and H. Darmon, The p-adic Birch and Swinnerton-Dyer conjecture, in preparation.

[3] J. Coates And A. Wiles, On the conjecture of Birch and Swinnerton-Dyer, Invent. Math. 39 (1977), 223-251.

[4] C.W. Curtis And I. Reiner, Methods of representation theory, Volume I, Wiley Interscience, New York, 1981.

[5] J. F. Humphreys, A course in group theory, Oxford University Press, 1996. 
[6] K. Ireland and M. Rosen, A classical introduction to modern number theory, Second Edition, Springer-Verlag, 1990.

[7] V. A. Kolyvagin, Finiteness of $E(\mathbb{Q})$ and $\operatorname{III}(\mathbb{Q})$ for a class of Weil curves, Math. USSR Izv. 32 (1989), 523-542.

[8] S. LANG, Algebra, Third Edition, Addison-Wesley, 1993.

[9] B. MazuR, Rational points on Abelian varieties in towers of number fields, Invent. Math., 18(1972), 183-266.

[10] B. Mazur and H.P.F. Swinnerton-Dyer, Arithmetic of Weil curves, Invent. Math., 25(1974), 1-61.

[11] M. R. Murty and V. K. Murty, Base change and the Birch- Swinnerton-Dyer conjecture, Contemp. Math. 143 (1993), 481-494.

[12] V. K. MurTy, Holomorphy of Artin $L$-functions, in: Proc. Ramanujan Centennial Intl. Conf. ed. R. Balakrishnan et. al., pp. 55-66, Ramanujan Math. Society, Madras, 1988.

[13] V. K. Murty, Class numbers of CM-fields with solvable normal closure, Compositio Math., to appear.

[14] M. V. NoRI, On subgroups of $\mathrm{GL}_{n}\left(\mathbf{F}_{p}\right)$, Invent. Math. 88 (1987), 257-275.

[15] E. Rees, Notes on Geometry, Springer-Verlag, 1983.

[16] D. RohrLich, The vanishing of certain Rankin-Selberg convolutions, in: Automorphic forms and analytic number theory, ed. R. Murty, pp. 123-133, CRM, Montréal, 1990.

[17] D. Rohrlich, Galois theory, elliptic curves, and root numbers, Comp. Math. 100 (1996) 311349 .

[18] K. Rubin, Elliptic curves with complex multiplication and the conjecture of Birch and Swinnerton-Dyer, Invent. Math., 64(1981), 455-470.

[19] J.-P. Serre, Linear Representations of Finite Groups, Springer-Verlag, 1977.

[20] J.-P. Serre, Propriétés galoisiennes des points d'ordre fini des courbes elliptiques, Invent. Math. 15 (1972), 259-331.

[21] J. H. Silverman, Advanced Topics in the Arithmetic of Elliptic Curves, Springer-Verlag, 1994.

[22] H. Stark, Some effective cases of the Brauer-Siegel theorem, Invent. Math. 23 (1974), 135-152.

Amir Akbary, Department of Mathematics and Statistics, Concordia University, 1455 de Maisonneuve Blvd. West, Montréal, Quebec, H3G 1M8, CANADA

E-mail: akbary@cicma.concordia.ca

V. Kumar Murty, Department of Mathematics, University of Toronto, 100 St. George Street, Toronto, Ontario, M5S 3G3, CANADA

E-mail: murty@math.toronto.edu 